\title{
The microbiota in the intestinal and respiratory tracts of naked mole-rats revealed by high-throughput sequencing
}

Wei Cong ${ }^{1+}$, Jin Xing ${ }^{2 \dagger}$, Yufang Feng ${ }^{2}$, Ji Wang ${ }^{2}$, Rui Fu², Bingfei Yue ${ }^{2}$, Zhengming He ${ }^{2}$, Lifang Lin ${ }^{1}$, Wenjing Yang ${ }^{1}$, Jishuai Cheng ${ }^{1}$, Wei Sun ${ }^{1}$ and Shufang Cui ${ }^{1 *}$ (D)

\begin{abstract}
Background: The naked mole-rat (NMR, Heterocephalus glaber) is being bred as a novel laboratory animal due to its unique biological characteristics, including longevity, cancer resistance, hypoxia tolerance, and pain insensitivity. It is expected that differences exist between the microbiota of wild NMRs and that of NMRs in an artificial environment. Overall, the effect of environment on changes in the NMR microbiota remains unknown. In an attempt to understand the microbiota composition of NMRs in captivity, variability in the microbiota of the intestinal and respiratory tracts of two groups of NMRs was assessed under two conditions.

Results: The results obtained by high-throughput sequencing revealed significant differences at the phylum, class, order, family and genus levels in the microbiota between the two groups of NMRs examined (first group in conventional environment, second group in barrier environment). For the trachea, 24 phyla and 533 genera and 26 phyla and 733 genera were identified for the first and second groups of animals. Regarding the cecum, 23 phyla and 385 genera and 25 phyla and 110 genera were identified in the microbiota of first and second groups of animals. There were no obvious differences between females and males or young and adult animals.

Conclusions: Our results suggest that the intestinal and respiratory tract NMR microbiota changed during captivity, which may be related to the transition to the breeding environment. Such changes in the microbiota of NMRs may have an effect on the original characteristics, which may be the direction of further research studies.
\end{abstract}

Keywords: Naked mole-rats, Microbiota, Cecum, Trachea, Bacterial diversity

\section{Background}

Naked mole-rats (NMRs, Heterocephalus glaber) are a unique species of rodents exhibiting high longevity, tumor resistance, hypoxia tolerance, and pain insensitivity [1-4]. Due to these attributes, NMRs are widely used as mammalian models in biomedical research studies investigating aging, cancer, and neurobiology as well as other topics. As a novel laboratory animal, the NMR is strongly differs from previously used experimental animals. However, as wild NMRs live in underground caves, which is a relatively low-oxygen and dark environment,

\footnotetext{
* Correspondence: youngstar_sf@163.com

+Wei Cong and Jin Xing contributed equally to this work.

${ }^{1}$ Laboratory Animal Centre, Second Military Medical University, No.8 Rd.

Panshan, Yangpu District, Shanghai, China

Full list of author information is available at the end of the article
}

a difference may exist between the microbiota of wild NMRs and those that live aboveground.

High-throughput sequencing has become an important method for studying the structure, diversity and pathogenicity of the bacterial flora of humans and animals [5-7]. Overall, the microbiota plays a significant role in maintaining the health and well-being of the mammalian host and also assists in nutrient metabolism $[8,9]$ and affects the body's immune function [10-12]. The microbiota is closely associated with the occurrence of tumors [13, 14], and the intestinal tract microbiota even exhibits antitumor immune effects [15]. As stated above, the NMR is characterized by longevity and anti-aging and cancer resistance. In an attempt to understand the composition of the NMR microbiota and its potential association with these properties, Debebe and colleagues $[16,17]$ preliminarily studied

(C) The Author(s). 2018 Open Access This article is distributed under the terms of the Creative Commons Attribution 4.0 International License (http://creativecommons.org/licenses/by/4.0/), which permits unrestricted use, distribution, and 
cultured bacteria from the bowels of wild NMRs by separating the gut flora and characterized the intestinal microbial ecosystem using next-generation sequencing. However, details regarding the microbiota of the intestinal and respiratory tracts of NMRs in captivity remain unknown. In this study, we sought to analyze the microbiota in the intestinal and respiratory tracts of NMRs in captivity in an effort to reveal the bacteria-related factors underlying the longevity of NMRs.

\section{Results}

\section{Sequencing results and acquisition of operational} taxonomic units (OTUs)

We assessed tracheal secretions and cecum contents of two groups of NMRs ( $n=33$ for the first; $n=30$ for the second) using high-throughput sequencing. The NMRs studied were derived from one closed colony obtained from Second Military Medical University [18]. The first group was bred in a conventional environment (fed without strict control and housed in an unsterilized cage); the second group was bred in a barrier environment (fed with strict control and caged with sterilization before use).

Approximately 1.5 million filtered sequences were obtained for tracheal secretions (approximately 580,000 for the first group and approximately 930,000 for the second group) and approximately 2 million filtered sequences for cecum contents (approximately 690,000 for the first group and approximately 1.03 million for the second group). After removing chimeric sequences and non-target regions, the number of residual sequences was, on average, 20,571 reads per sample.

All sequences were classified for OTU analysis according to the similarity threshold setting (0.97). The tracheal results for the first group showed 24,115 OTUs, with an average of 2445 in each animal and an average of 421-bp high-quality sequences (the shortest sequence was $200 \mathrm{bp}$; the longest sequence was $451 \mathrm{bp}$ ). For the second group, 5470 OTUs, with an average of $396 \mathrm{bp}$ (the shortest was $200 \mathrm{bp}$; the longest was $462 \mathrm{bp}$ ) were acquired by sequencing.

The number of OTUs in the first and second groups fit a normal distribution, and the difference in the number of OTUs was statistically significant $(P<0.001)$. For the first group, there was no significant difference in the number of OTUs $(P=0.224)$ between male and female NMRs; similarly, no significant difference in the number of OTUs $(P=0.074)$ between young $(\leq 20$ weeks $)$ and adult (> 20 weeks) NMRs in was observed. Although the number of OTUs was not significantly different $(P=0.263)$ between male and female animals in the second group, there was a significant difference $(P=0.009)$ between young and adult animals.

Sequencing of the cecal contents in the first group resulted in 110,815 OTUs, with an average of 3358 OTUs per animal and an average read length of $416 \mathrm{bp}$ (the shortest was $200 \mathrm{bp}$; the longest was $447 \mathrm{bp}$ ). A total of 9118 OTUs were sequenced for the second group of 30 NMRs, with an average read length of $385 \mathrm{bp}$ (the shortest was $201 \mathrm{bp}$; the longest was $462 \mathrm{bp}$ ).

OTUs for the cecal contents were normally distributed and showed a significant difference $(P<0.001)$ between the first and second groups. There was no significant difference in the number of cecal OTUs $(P=0.479)$ between male and female NMRs and no significant difference in the number of cecal OTUs $(P=0.499)$ between the two age groups in the first group. In addition, the number of cecal OTUs was not significantly different $(P=0.263)$ between male and female animals or between young and adult animals $(P=0.685)$ in the second group.

The sequencing data were analyzed by alpha diversity analysis, including the coverage of each sample library, Shannon index, Chaol index, ACE index, and Simpson index (Table 1), and MOTHUR was used for rarefaction curve analysis (Fig. 1a, b, c, d). According to the rarefaction curves, there was no significant change in the number of OTUs, and the number of sequences was able to reflect the diversity in the ileocecum microbiota.

\section{LEfSe (linear discriminant analysis effect size) analysis}

In LEfSe analysis, differences between the two groups of NMRs indicated significant differences in the microbiota at the phylum, class, order, family and genus levels. For the trachea and cecum microbiota, $117(65+52)$ and 93 $(59+34)$ differentially abundant taxonomic clades, respectively, were obtained for the two groups of animals. LDA (linear discriminant analysis) scores were all higher than 2.4 (data not shown). The most significantly different taxa in the trachea were Enterobacteriaceae (Proteobacteria) in the first group and Pseudomonadaceae (Proteobacteria) in the second group. Burkholderia, Neisseria, Pasteurella, Staphylococcus and Streptococcus were clearly present in the trachea of the first group of animals but were effectively eliminated in the second group (Fig. 2a). Regarding the cecum microbiota, the greatest differences were exhibited by Bacteroidales (Bacteroidetes) in the first groups and Ruminococcaceae (Firmicutes) in the second. It was clear that Bacteroidetes and Proteobacteria accounted for a significant portion in the first group of animals, whereas Citrobacter and Klebsiella were controlled in the second group. Bacillus (Firmicutes) and Spirochaetia (Spirochaetes) were identified according to their characteristics (Fig. 2b).

\section{Comparison of microbiota at the phylum level}

Overall, the results of microbiota analysis at the phylum level for the first groups of endotracheal samples demonstrated that of the 24 phyla identified, the content of 
Table 1 Number of sequences retrieved, number of OTUs and alpha diversity indexes

\begin{tabular}{|c|c|c|c|c|}
\hline \multirow[t]{2}{*}{ Variable (Average) } & \multicolumn{2}{|l|}{ Trachea } & \multicolumn{2}{|l|}{ Cecum } \\
\hline & 1st collection & 2nd collection & 1st collection & 2nd collection \\
\hline Sequence number & $17,560 \pm 1786$ & $30,872 \pm 6892$ & $20,571 \pm 2280$ & $34,470 \pm 4870$ \\
\hline OTUs & $2445 \pm 790$ & $304 \pm 74$ & $3358 \pm 887$ & $304 \pm 48$ \\
\hline Coverage & $0.915 \pm 0.028$ & $0.995 \pm 0.001$ & $0.893 \pm 0.037$ & $0.997 \pm 0.001$ \\
\hline Shannon & $4.85 \pm 0.77$ & $2.67 \pm 0.19$ & $5.61 \pm 0.73$ & $3.17 \pm 0.48$ \\
\hline Chaol & $5386.31 \pm 1761.64$ & $610.22 \pm 141.98$ & $8830.93 \pm 2567.26$ & $592.40 \pm 122.48$ \\
\hline ACE & $8259.34 \pm 2864.72$ & $918.44 \pm 238.74$ & $13,870.80 \pm 4780.53$ & $674.65 \pm 151.16$ \\
\hline Simpson & $0.075 \pm 0.070$ & $0.136 \pm 0.026$ & $0.034 \pm 0.031$ & $0.146 \pm 0.088$ \\
\hline
\end{tabular}

Proteobacteria $(66.90 \%)$ was the highest, followed by Firmicutes (18.32\%), Bacteroidetes (10.10\%), Fusobacteria $(2.73 \%)$, unclassified (1.84\%), Spirochaetes $(0.06 \%)$ and Planctomycetes $(0.01 \%)$. In the second group of animals, the microbiota comprised 26 phyla, with the highest levels for Proteobacteria (47.33\%) and Firmicutes (41.91\%), which accounted for nearly $90 \%$ of the population, followed by Bacteroidetes (2.97\%), Actinobacteria
(2.43\%), unclassified (3.51\%), Fusobacteria (0.18\%) and Spirochaetes $(0.17 \%)$.

The trachea microbiota of the two groups of animals differed at the phylum level (Fig. 3a). Although Verrucomicrobia $(P<0.001)$ and Nitrospirae $(P=0.028)$ were not found in the second group, Actinobacteria $(P<0.001)$, Candidate_division_TM7 $(P<0.001)$, Acidobacteria $(P<$ $0.001)$ and Crenarchaeota $(P=0.294$, only 1 animal $)$ were

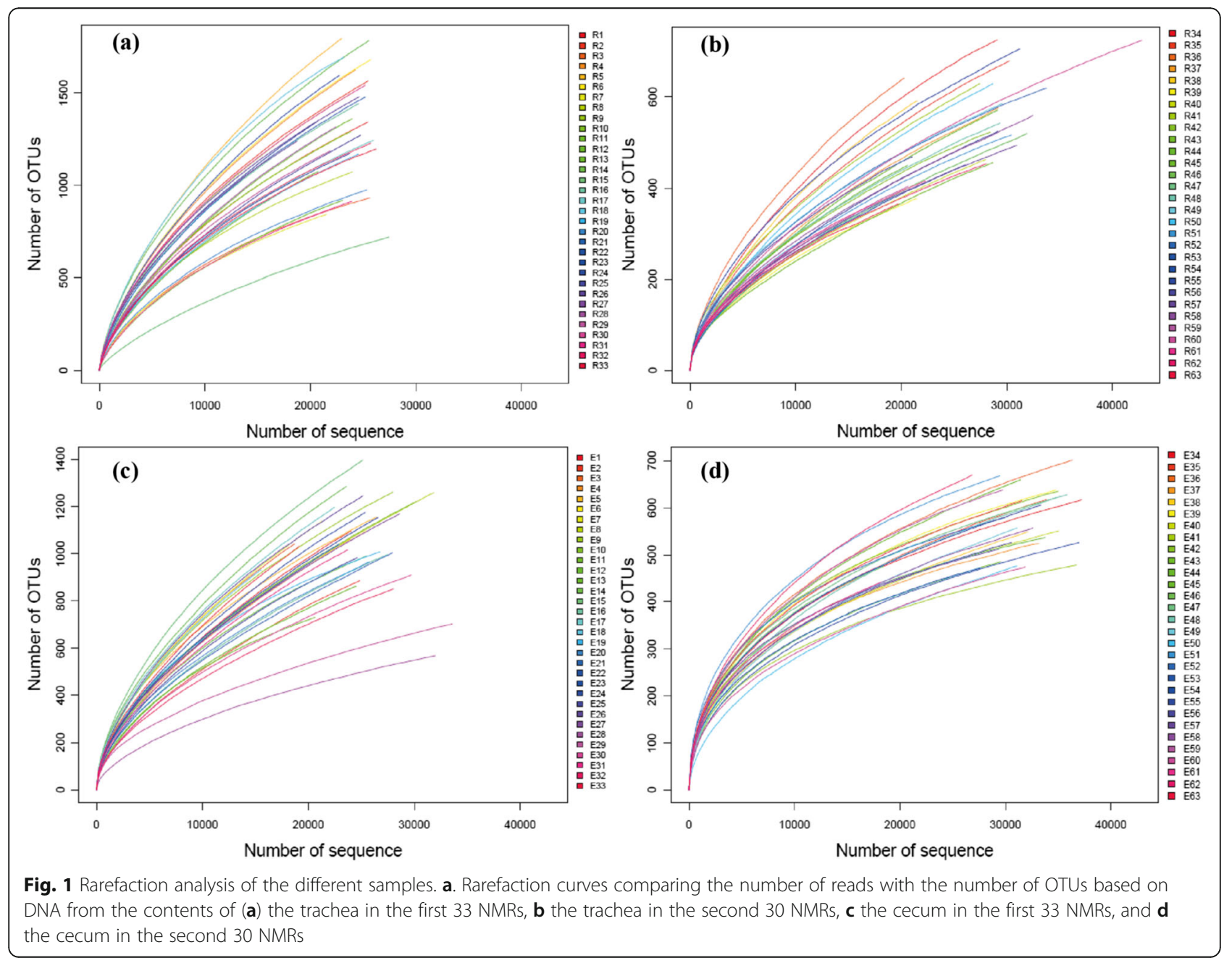




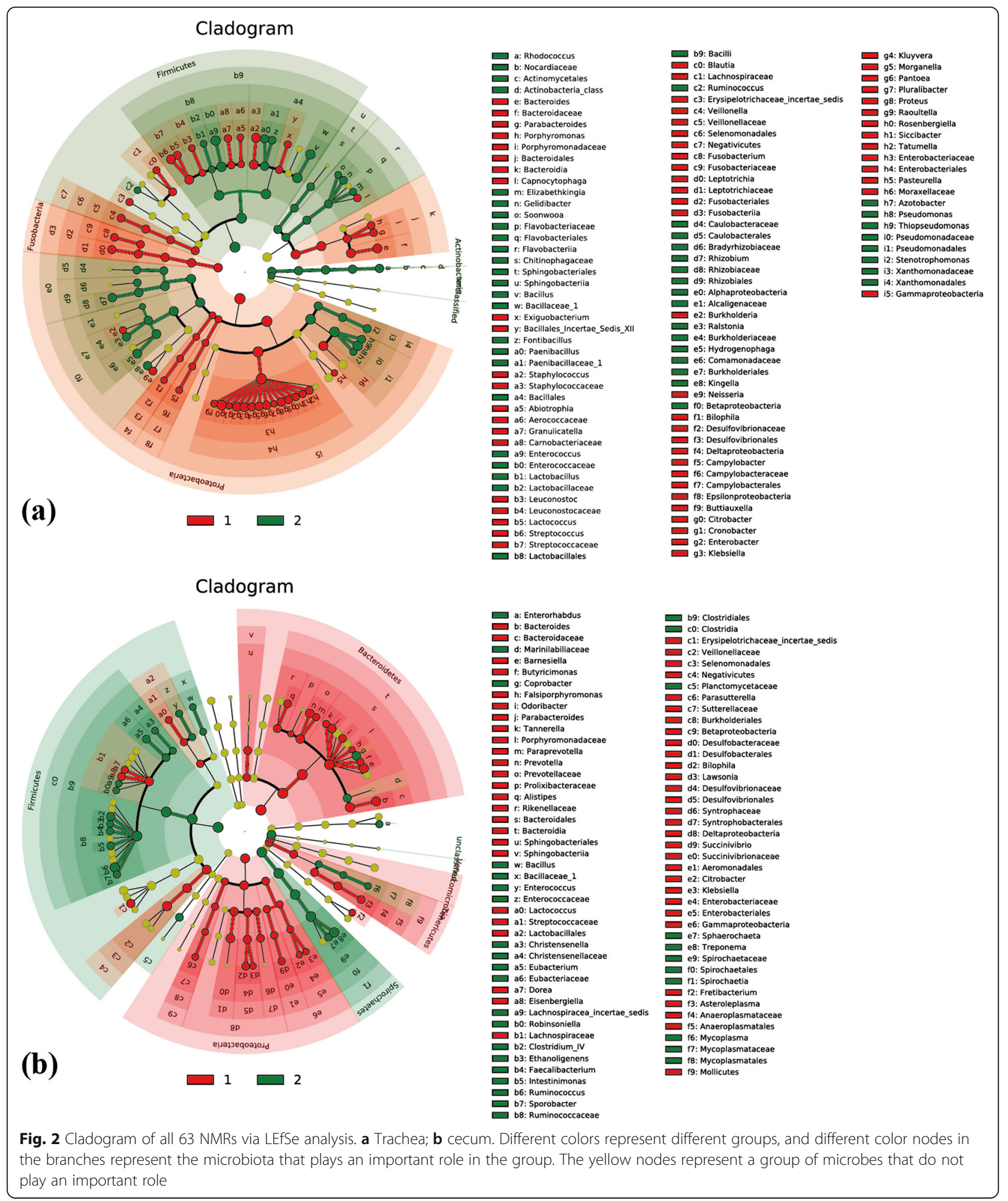




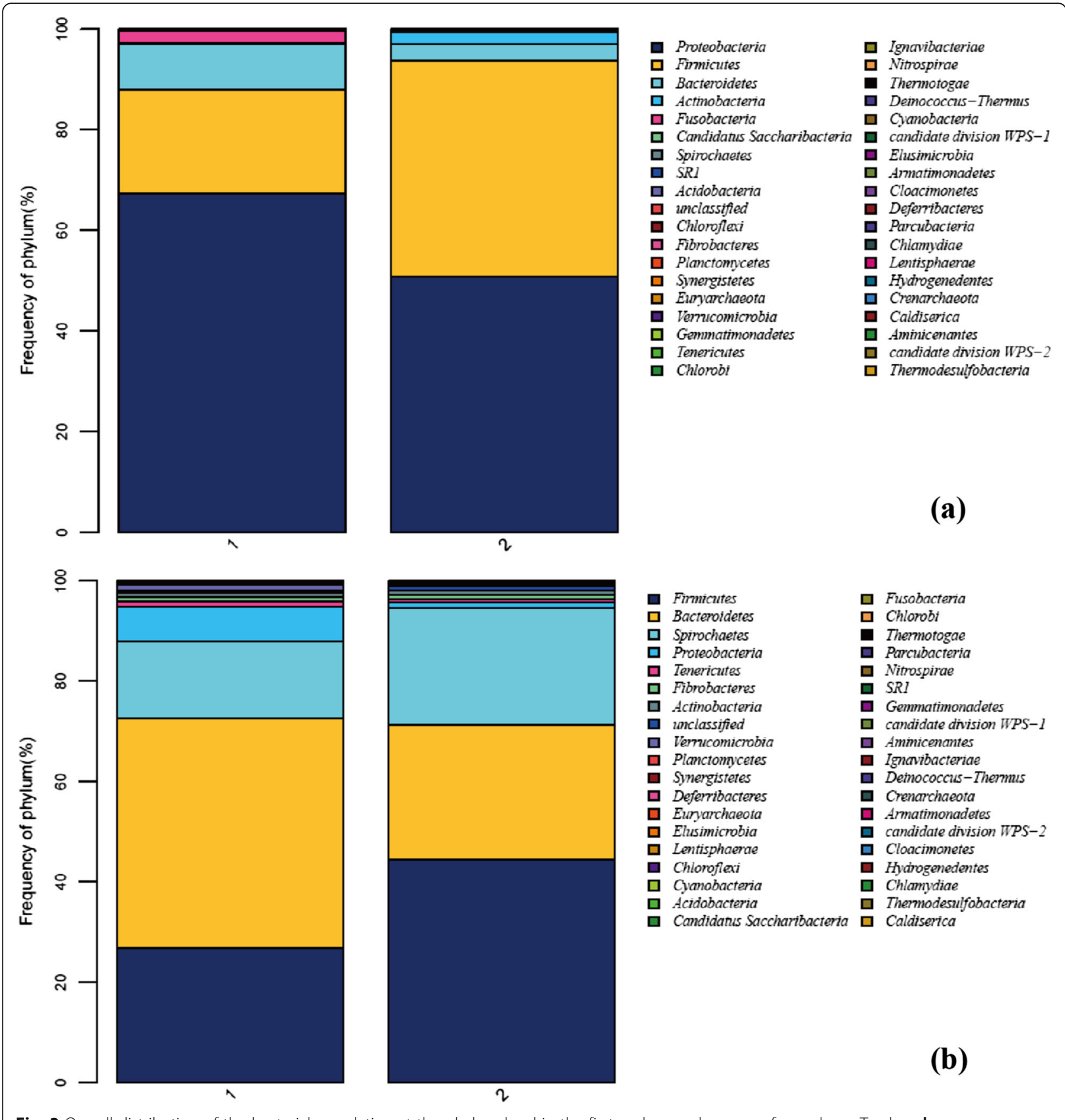

Fig. 3 Overall distribution of the bacterial population at the phylum level in the first and second groups of samples. a Trachea; $\mathbf{b}$ cecum

increased in abundance. Variation between the groups was significant with regard to Proteobacteria, Firmicutes, Fusobacteria, Planctomycetes, Chloroflexi, and Euryarchaeota $(P<0.001)$, as well as Cyanobacteria $(P=0.032)$, Chlorobi $(P=0.030)$, and Gemmatimonadetes $(P=0.001)$. Moreover, Chloroflexi, Synergistetes, Deinococcus, Deferribacteres, Lentisphaerae, Armatimonadetes and Actinobacteria were different between young and adult NMRs $(P<0.05)$. Conversely, no differences in bacterial taxa were observed between male and female NMRs (Table 2).
For the first group, 23 phyla were found among the cecum contents, including the highest content of Bacteroidetes $(40.56 \%)$, followed by Firmicutes (26.21\%), Spirochaetes (15.43\%), unclassified (8.16\%), Proteobacteria (4.58\%), Verrucomicrobia (1.06\%), and Fibrobacteres (0.74\%). Although Verrucomicrobia accounted for only $1.06 \%$ of the abundance, it was the only taxon with a higher content in male young animals (10 to 20 weeks). The cecum microbiota of the second group consisted of 25 phyla, including the highest content of Firmicutes (45.81\%), 
Table 2 Phyla ratio in the trachea in time, sex, and age

\begin{tabular}{|c|c|c|c|c|c|c|c|c|}
\hline \multirow[t]{3}{*}{ Phylum } & \multicolumn{4}{|l|}{ First ratio } & \multicolumn{4}{|l|}{ Second ratio } \\
\hline & \multicolumn{2}{|l|}{ Female } & \multicolumn{2}{|l|}{ Male } & \multicolumn{2}{|l|}{ Female } & \multicolumn{2}{|l|}{ Male } \\
\hline & $10 \sim 20$ weeks & $20 \sim 35$ weeks & $10 \sim 20$ weeks & $20 \sim 35$ weeks & $10 \sim 20$ weeks & $20 \sim 35$ weeks & $10 \sim 20$ weeks & $20 \sim 35$ weeks \\
\hline Proteobacteria & 74.73 & 55.66 & 59.26 & 77.965 & 53.51 & 48.18 & 42.03 & 45.61 \\
\hline Firmicutes & 16.03 & 19.73 & 22.66 & 14.865 & 36.18 & 44.98 & 43.62 & 42.87 \\
\hline Bacteroidetes & 5.73 & 17.88 & 12.50 & 4.275 & 3.45 & 2.74 & 2.77 & 2.93 \\
\hline Fusobacteria & 1.51 & 4.01 & 3.46 & 1.93 & 0.16 & 0.10 & 0.14 & 0.30 \\
\hline unclassified & 1.89 & 2.51 & 2.01 & 0.93 & 4.06 & 0.49 & 3.95 & 5.54 \\
\hline Spirochaetes & 0.04 & 0.11 & 0.07 & 0.025 & 0.08 & 0.46 & 0.03 & 0.09 \\
\hline Planctomycetes & 0.02 & 0.02 & 0.01 & 0.005 & 0.02 & 0.04 & 0.01 & 0.05 \\
\hline Chloroflexi & 0.01 & 0.02 & 0.01 & / & 0.02 & 0.05 & 0.03 & 0.05 \\
\hline Verrucomicrobia & 0.01 & 0.01 & 0.01 & / & / & / & / & / \\
\hline Synergistetes & $>0.01$ & 0.01 & 0.01 & / & / & 0.03 & 0.01 & 0.01 \\
\hline Cyanobacteria & 0.01 & 0.01 & $>0.01$ & / & / & $>0.01$ & $>0.01$ & $>0.01$ \\
\hline Chlorobi & 0.01 & / & $>0.01$ & / & $>0.01$ & 0.01 & $>0.01$ & 0.01 \\
\hline Thermotogae & 0.01 & / & $>0.01$ & / & $>0.01$ & $>0.01$ & / & / \\
\hline Nitrospirae & $>0.01$ & 0.01 & $>0.01$ & / & / & / & / & / \\
\hline Tenericutes & / & 0.01 & $>0.01$ & I & $>0.01$ & $>0.01$ & / & / \\
\hline Gemmatimonadetes & $>0.01$ & 0.01 & $>0.01$ & l & 0.01 & 0.01 & 0.02 & 0.01 \\
\hline Euryarchaeota & $>0.01$ & $>0.01$ & $>0.01$ & / & 0.01 & 0.02 & 0.01 & 0.02 \\
\hline Fibrobacteres & $>0.01$ & 0.01 & / & 0.005 & 0.01 & 0.06 & $>0.01$ & 0.04 \\
\hline Deinococcus-Thermus & $>0.01$ & / & $>0.01$ & 0.005 & / & 0.01 & $>0.01$ & $>0.01$ \\
\hline Chlamydiae & $>0.01$ & $>0.01$ & $>0.01$ & / & / & $>0.01$ & $>0.01$ & $>0.01$ \\
\hline Elusimicrobia & / & $>0.01$ & / & / & / & / & / & $>0.01$ \\
\hline Deferribacteres & / & $>0.01$ & / & / & / & 0.01 & / & / \\
\hline Lentisphaerae & / & $>0.01$ & / & / & $>0.01$ & & / & $>0.01$ \\
\hline Armatimonadetes & / & $>0.01$ & / & / & / & $>0.01$ & / & $>0.01$ \\
\hline Actinobacteria & / & / & / & / & 2.40 & 2.70 & 2.25 & 2.38 \\
\hline Candidate_division_TM7 & / & / & / & / & 0.04 & 0.04 & 0.08 & 0.02 \\
\hline Acidobacteria & / & / & / & / & 0.02 & 0.04 & 0.02 & 0.03 \\
\hline Crenarchaeota & / & / & / & / & / & / & / & $>0.01$ \\
\hline
\end{tabular}

followed by Bacteroidetes (24.08\%), Spirochaetes (23.38\%), unclassified (2.61\%), Proteobacteria (1.46\%), Fibrobacteres $(0.89 \%)$ and Actinobacteria (0.69\%).

Differences in diversity at the phylum level were also found for the cecum contents between the two groups (Fig. 3b). Compared with the first group, Bacteroidetes in the second was markedly decreased $(P<0.001)$; Firmicutes $(P=0.026)$, Spirochaetes $(P=0.016)$, Tenericutes $(P<$ $0.001)$ and Planctomycetes $(P<0.001)$ were also significantly increased and Proteobacteria $(P<0.001)$, Verrucomicrobia $(P<0.001)$ and unclassified $(P<0.001)$ significantly reduced. Deinococcus-Thermus, Actinobacteria, Acidobacteria, Candidate_division_TM7 and Chlamydiae were only present in the second group of animals, whereas Thermotogae and Thermodesulfobacteria, which were low in abundance in the first group of animals, were absent from the cecum microbiota in the second groups of animals. Additionally, Nitrospirae, Deinococcus-Thermus and Chlamydiae were rare in the second group. Regarding age, the phyla in young (10 to 20 weeks) and adult (20 to 35 weeks) animals only differed for Planctomycetes $(P=0.013)$ and Actinobacteria $(P=0.018)$; regarding gender, only Fusobacteria $(P=0.024)$ differed (Table 3$)$.

\section{Comparison of microbiota at the genus level}

Based on the analysis at the genus level, the microbiota was more abundant in the trachea samples from the second group (Fig. 4a), whereas the results for the cecum (Fig. 4b) samples were the opposite.

The trachea samples from the first group comprised 533 genera, the main genus (content percentage $>1 \%$ ) of which consists of 15 genera. The percentage of Enterobacter was 
Table 3 Phyla ratio in the cecum in time, sex, and age

\begin{tabular}{|c|c|c|c|c|c|c|c|c|}
\hline \multirow[t]{3}{*}{ Phylum and OTUs } & \multicolumn{4}{|l|}{ First ratio } & \multicolumn{4}{|l|}{ Second ratio } \\
\hline & \multicolumn{2}{|l|}{ Female } & \multicolumn{2}{|l|}{ Male } & \multicolumn{2}{|l|}{ Female } & \multicolumn{2}{|l|}{ Male } \\
\hline & 10 20 weeks & $20 \sim 35$ weeks & $10 \sim 20$ weeks & $20 \sim 35$ weeks & $10 \sim 20$ weeks & $20 \sim 35$ weeks & $10 \sim 20$ weeks & $20 \sim 35$ weeks \\
\hline Bacteroidetes & 38.16 & 47.73 & 40.37 & 35.97 & 21.35 & 22.36 & 20.79 & 32.12 \\
\hline Firmicutes & 29.38 & 20.00 & 30.50 & 24.97 & 34.51 & 52.51 & 48.60 & 47.84 \\
\hline Spirochaetes & 16.85 & 17.46 & 10.99 & 28.43 & 38.25 & 14.96 & 24.40 & 13.40 \\
\hline Unclassified & 7.79 & 8.85 & 8.99 & 7.01 & 2.66 & 2.91 & 3.30 & 1.43 \\
\hline Proteobacteria & 5.17 & 5.03 & 4.87 & 3.25 & 1.36 & 1.58 & 1.45 & 1.66 \\
\hline Verrucomicrobia & 0.01 & $<0.01$ & 3.17 & 0.01 & 0.17 & 0.03 & 0.05 & 0.08 \\
\hline Fibrobacteres & 2.01 & 0.51 & 0.45 & 0.02 & 0.08 & 3.19 & 0.16 & 1.53 \\
\hline Synergistetes & 0.28 & 0.11 & 0.28 & 0.08 & 0.30 & 0.32 & 0.13 & 0.25 \\
\hline Deferribacteres & 0.20 & 0.23 & 0.12 & 0.24 & 0.22 & 0.27 & 0.16 & 0.11 \\
\hline Tenericutes & 0.03 & 0.03 & 0.11 & 0.02 & 0.09 & 0.10 & 0.21 & 0.31 \\
\hline Euryarchaeota & 0.08 & 0.03 & 0.05 & 0.01 & 0.01 & 0.01 & 0.02 & 0.02 \\
\hline Elusimicrobia & 0.03 & 0.02 & 0.05 & 0.01 & 0.04 & 0.01 & 0.03 & 0.01 \\
\hline Lentisphaerae & 0.01 & 0.01 & 0.03 & / & 0.02 & 0.01 & $<0.01$ & 0.01 \\
\hline Chloroflexi & 0.01 & 0.01 & 0.01 & $<0.01$ & 0.03 & 0.01 & 0.01 & 0.01 \\
\hline Planctomycetes & 0.01 & / & $<0.01$ & $<0.01$ & 0.27 & 0.50 & 0.19 & 0.43 \\
\hline Gemmatimonadetes & $<0.01$ & / & $<0.01$ & $<0.01$ & $<0.01$ & / & / & / \\
\hline Cyanobacteria & $<0.01$ & / & $<0.01$ & / & 0.01 & $<0.01$ & $<0.01$ & / \\
\hline Thermotogae & / & / & $<0.01$ & / & / & / & / & / \\
\hline Nitrospirae & $<0.01$ & / & / & 0.01 & / & / & $<0.01$ & / \\
\hline Chlorobi & $<0.01$ & / & / & / & / & 0.01 & / & / \\
\hline Fusobacteria & $<0.01$ & / & / & / & $<0.01$ & $<0.01$ & I & / \\
\hline Thermodesulfobacteria & $<0.01$ & / & / & / & / & / & / & / \\
\hline Deinococcus-Thermus & / & / & / & / & / & $<0.01$ & / & / \\
\hline Actinobacteria & / & / & / & / & 0.63 & 1.23 & 0.47 & 0.76 \\
\hline Acidobacteria & / & / & / & / & 0.01 & / & $<0.01$ & $<0.01$ \\
\hline Candidate_division_TM7 & / & / & / & / & $<0.01$ & $<0.01$ & $<0.01$ & $<0.01$ \\
\hline Chlamydiae & / & / & / & / & $<0.01$ & / & / & / \\
\hline
\end{tabular}

the highest, followed by Staphylococcus, Bacteroides, Klebsiella, Pantoea and Acinetobacter. There were 733 genera in the trachea samples from the second group, the main genus (content percentage $>1 \%$ ) of which had 11. Bacillus and Pseudomonas accounted for more than half of all bacteria. The next most abundant taxon was unclassified, followed by Haemophilus, Rhizobium and Paenibacillus.

Regarding the cecum samples from the first group, 385 genera were identified, the contents of which were all low. The main genus had (>1\%) 21. The percentage of Treponema was the highest, followed by unclassified-Porphyromonadaceae, Bacteroides, unclassified and Allobaculum. In total, 110 genera were identified in the cecum samples from the second group, the main genus (content percentage $>1 \%$ ) of which had 16 . On the very top of the list were Treponema, Ruminococcus,
unclassified-Porphyromonadaceae, Allobaculum and Inertae-Sedis.

NMDS (nonmetric multidimensional scaling) analysis

NMDS analysis of the trachea and cecum sequencing results (Fig. 5a, b) revealed a basically concentrated bacterial structure for the groups. Regardless of whether the samples were obtained from the respiratory or intestinal tract, the two batches of bacteria differed significantly. There were no obvious differences between females and males or young and adult animals. The findings also showed that the microbiota of the NMR trachea and intestinal tract tended to be stable after artificial feeding. However, after breeding, the microbiota in the cecum became polarized, with the microbiota in the same aged animals tending to be the same. 

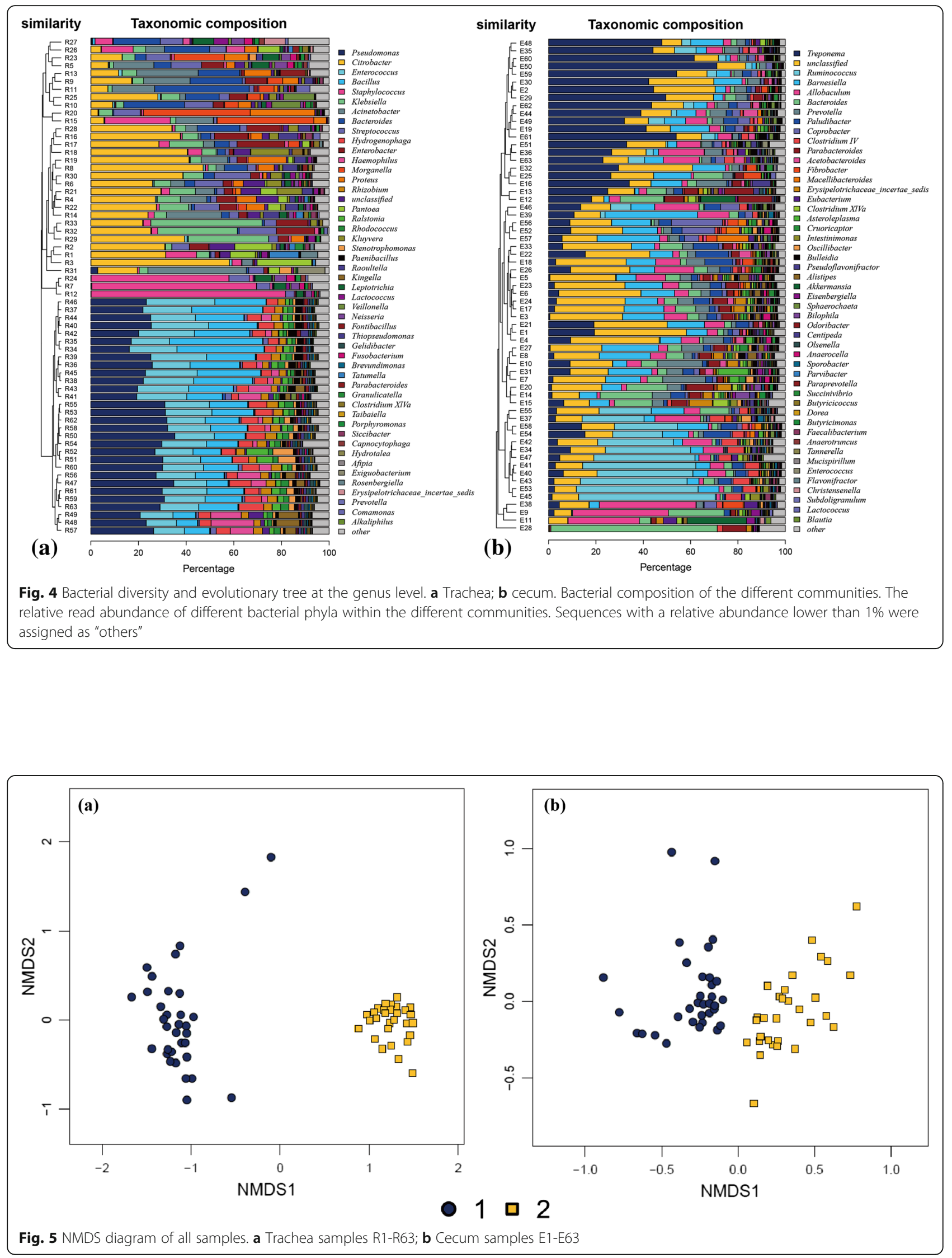


\section{Pathogens}

The pathogens or conditioned pathogens identified in the trachea samples from the first group were Klebsiella pneumoniae, Citrobacter freundii [19], Enterobacter cloacae [20], Proteus vulgaris, Proteus mirabilis, Staphylococcus sciuri, Klebsiella oxytoca, Aeromonas caviae, Streptococcus suis and Mycoplasma pulmonis. The main pathogen in the cecum samples from the first group was Klebsiella pneumoniae.

Spirochaetaceae was always present in the cecum samples from the two groups of animals. Mycoplasma was present in cecum samples from the second group but not in the trachea samples, a result that may be due to residual nucleic acid.

\section{Discussion}

For the first time, the microbiota in the trachea and cecum of NMRs in captivity was investigated using Illumina MiSeq high-throughput sequencing, and our results revealed the bacterial diversity of these two organs at the phylum and genus levels. We demonstrated that in the first group of animals, the most abundant taxon was Bacteroidetes (40.56\%) in cecum samples, followed by Firmicutes (26.21\%), which was similar to the result reported by Debebe [16] in which Bacillus megaterium accounted for $45.2 \%$ of the culture, followed by Bacteroides thetaiotaomicron (19.4\%). We also isolated cultured bacteria from the cecum contents and performed $16 \mathrm{~S}$ rDNA sequencing (Table 4). The main bacteria isolated from the cecum samples from the first group were Firmicutes (18 species), Bacteroidetes (10 species) and Proteobacteria (17 species); Actinobacteria and Ascomycota were the main normal microflora in the wild NMR cecum. Klebsiella pneumonia and Bacillus pumilus were isolated from the colon instead of the cecum in wild NMRs. The microflora changed in the second group of animals, which included Firmicutes (19 species), Bacteroidetes (1 species), Proteobacteria (3 species), and Actinobacteria (1 species). Notably, a significant reduction in Bacteroidetes was observed in the second group of animals, which was consistent with the result of high-throughput sequencing in which the content of Bacteroidetes was reduced by almost twofold (Fig. 2). In the same case, only 3 species of Proteobacteria were observed. The results of high-throughput sequencing showed that the level of Proteobacteria increased in the second group, which suggested that the diversity among Proteobacteria strains decreased, and that the contents of the remaining strains increased. The NMRs in the present study were different from the wild animals examined by Debebe; although the main types of bacteria were similar, the strains were significantly different. This inconsistency may be related to the transition from the wild environment to the artificial breeding environment. The findings showed that the first group of
NMRs maintained the microorganisms present in the original wild state. Sequencing was used to reconstruct the changes microbiota in NMRs from wild to laboratory animals.

\section{The respiratory tract}

Studies have shown that microbial diversity in the lung is significantly lower in patients with asthma, chronic obstructive pulmonary disease (COPD) and cystic fibrosis than in healthy individuals, mainly manifested by the increase in Proteobacteria (such as Haemophilus) or Firmicutes (such as Staphylococcus) [21]. Proteobacteria (66.90\%), Firmicutes (18.32\%), Bacteroidetes (10.10\%) and Fusobacteria (2.73\%) accounted for more than $98 \%$ of the microbiota in the trachea samples from the second group of NMRs. Analyses of human lung microbiota have revealed that there is a lower risk of later disease with great diversity in early exposure [22]. However, it is unknown whether NMRs conform to this generality. Moreover, host genes influence the upper airway microbial composition in humans and affect mucosal immunity [23]. Thus, our findings suggest that the natural anticancer properties of NMRs are largely related to their genetic properties.

Chen et al. [24] compared the microbial diversity of healthy individuals and patients with dental caries and periodontal disease and identified Firmicutes, Bacteroidetes, Proteobacteria, Actinobacteria and Fusobacteria in healthy individuals, which is similar to the microbiota observed in the NMR trachea. Interestingly, the observed microbiota changes in the trachea of NMRs in captivity (Firmicutes 18 to $42 \%$, Proteobacteria 67 to $47 \%$ ) were similar to the changes observed in oral bacteria in periodontitis patients (Firmicutes 36 to 60\%, Proteobacteria 50 to 25\%). Xiao et al. [25] demonstrated in rats that the microbial community in the oral cavity can be affected by an altitude-induced hypoxic environment, with more severe periodontal lesions. Therefore, it is necessary to investigate bacteria in other respiratory areas, such as the oral cavity, which may serve as a good model for oral disease studies.

The microbiota in the respiratory tract, which are more likely to be influenced by the environment, appear to not be as influential as those in the gut. Our sequencing results also showed that the bacteria in the respiratory samples from the second group of animals were significantly different from those in the first group. The number of genera increased by one-third, and the first several dominant genera were all different. This may be related to exposure to some bacteria that cannot be cleared by the body in the new environment and because microbiota of the respiratory tract were adapted to this new environment. The respiratory tract is a site of microbial entry, and these bacteria are a major factor in the maintenance of respiratory physiology and immune balance in animals [26]. 
Table 4 Distribution of cultured microbiota in the cecum

\begin{tabular}{|c|c|c|c|}
\hline Phylum & 1st collection & 2nd collection & Reference [16] \\
\hline \multirow[t]{19}{*}{ Firmicutes } & Allobaculum stercoricanis & Bacillus licheniformis & Bacillus megaterium \\
\hline & Bacillus altitudinis & Bacillus megaterium & Staphylococcus gallinarum \\
\hline & Bacillus aryabhattai & Bacillus pumilus & Staphylococcus xylosus \\
\hline & Bacillus cereus & Bacillus subtilis & Staphylococcus sciuri \\
\hline & Bacillus licheniformis & Bacillus velezensis & Paenibacillus spp. \\
\hline & Bacillus megaterium & Brevibacillus laterosporus & Staphylococcus warneri \\
\hline & Bacillus pumilus & Enterococcus sp. & Brevibacillus spp. \\
\hline & Bacillus safensis & Lactobacillus animalis & Lysinibacillus fusiformis \\
\hline & Bacillus subtilis & Lactococcus lactis & streptococcus mitis/oralis \\
\hline & Eubacterium cylindroides & Paenibacillus amylolyticus & Enterococcus casseliflavus \\
\hline & Eubacterium limosum & Paenibacillus glucanolyticus & \\
\hline & Lysinibacillus sp. & Paenibacillus pabuli & \\
\hline & Paenibacillus terrigena & Staphylococcus cohnii & \\
\hline & Staphylococcus cohnii & Staphylococcus haemolyticus & \\
\hline & Staphylococcus gallinarum & Staphylococcus kloosii & \\
\hline & Staphylococcus kloosii & Staphylococcus pettenkoferi & \\
\hline & Staphylococcus sciuri & Staphylococcus saprophyticus & \\
\hline & Staphylococcus sp. & Staphylococcus warneri & \\
\hline & & Streptococcus salivarius & \\
\hline \multirow[t]{7}{*}{ Bacteroidetes } & Virgibacillus sp. & Bacteroides thetaiotaomicron & Bacteroides ovatus \\
\hline & Alistipes shahii & & Bacteroides thetaiotaomicron \\
\hline & Bacteroidaceae bacterium & & Bacteroides fragilis \\
\hline & Bacteroides fragilis & & Bacteroided vulgatus \\
\hline & Bacteroides stercorirosoris & & \\
\hline & Bacteroides thetaiotaomicron & & \\
\hline & Bacteroides xylanisolven & & \\
\hline \multirow[t]{15}{*}{ Proteobacteria } & Parabacteroides distasonis & Proteus penneri & Enterobacter cloacae/osurae \\
\hline & Acinetobacter baumannii & Pseudomonas fragi & \\
\hline & Citrobacter freundii & & \\
\hline & Enterobacter aerogenes & & \\
\hline & Enterobacter asburiae & & \\
\hline & Enterobacter cloacae & & \\
\hline & Enterobacter hormaechei & & \\
\hline & Klebsiella pneumoniae & & \\
\hline & Klebsiella variicola & & \\
\hline & Lactobacillus plantarum & & \\
\hline & Lonepinella & & \\
\hline & Morganella morganii & & \\
\hline & Pantoea agglomerans & & \\
\hline & Proteus penneri & & \\
\hline & Proteus vulgaris & & \\
\hline Actinobacteria & & Microbacterium sp. & \\
\hline
\end{tabular}


The results of tracheal sequencing confirmed a certain proportion of Pasteurella, though the distribution bar plot figure generated by the software utilized did not list this genus (Fig. 4a); only Haemophilus was listed, which is likely due to the similarity in $16 \mathrm{~s}$ rRNA sequence [27]. Pasteurella is one of the most common genera in laboratory animals, and the species that have the greatest influence on laboratory animals are $P$. multocida and $P$. pneumotropica. These pathogens were not found in the NMRs in our study.

\section{The cecum}

The results of high-throughput sequencing also confirmed that Firmicutes and Bacteroidetes are predominant bacteria in the gut microbiota. However, unlike the normal human gut flora, the abundance of Firmicutes was $3 / 5$ that of Bacteroidetes in the first group of animals and $2 / 1$ in the second group, which was very different from previous reports [16, 28]. This finding provides new data regarding the range of normal bacteria in the NMR.

A previous study [29] reported that changes in Bacteroides, Parabacteroides, Alistipes and Akkermansia in the gut microbiota are closely related to the occurrence of colorectal cancer (CRC). The blocking effect of the antibody targeting cytotoxic $\mathrm{T}$ lymphocyte antigen 4 (CTLA-4) depends on the types of Bacteroides in the body [13]. Bacteroides in NMRs in the cecum samples from the first group of accounted for $40.56 \%$ and decreased to $24.08 \%$ in the second group. These changes, including other flora, such as Lactococcus, can effectively inhibit the growth of cancer cells [30]. The effects of microbiota on the health and longevity of NMRs require long-term observations and further studies.

The main pathogen found in NMR was Klebsiella pneumoniae; moreover, it is worth noting that the highest genus content in the cecum was Treponema (Spirochaetaceae), which is also an important pathogen. This situation is consistent with microscopy sampling., which revealed a large number of seagull or spiral-shape long bacteria in the cecum contents. LEfSe analysis showed that Spirochaetaceae can serve as a biomarker after a period of breeding. Although the carrier rate of Treponema decreased after breeding, the contents of Treponema in most animals increased. This change may be related to the difference in breeding environment, as Debebe [17] noted that Treponema species increase the ability of NMRs to digest and extract valuable nutrition from fibrous naturally occurring plants, which are enriched in the NMR diet.

\section{Conclusions}

This study laid the foundation for the laboratory animalization of this new experimental animal. The
NMR microbiota composition is potentially associated with its breeding environment. The factors associated with NMR longevity may lie in the microbiota in the intestinal tract. Our results show that the microbiota of NMRs differs between the wild environment and the artificial breeding environment. The artificial breeding of NMR is intended to make better use of its antitumor and longevity properties. In captivity, changes in an animal's microbiota may have an effect on its original characteristics, which may be the subject of further studies.

\section{Methods}

\section{Sample collection}

NMRs were obtained from the Laboratory Animal Center of the Second Military Medical University. The animal handling and study procedures were in accordance with the current Chinese regulation "GB14925- 2010 Laboratory animal requirements of environment and housing facilities" (Chinese version). The complete protocol was reviewed and approved by the Institutional Animal Care and Use Committee of the Second Military Medical University. The trachea and cecum were collected from 63 animals, which were sampled at random. Approximately $0.5 \mathrm{~cm}$ of the middle section of the trachea was removed using ophthalmic scissors and stored in sterile PBS in Corning tubes under aseptic conditions. Cecum samples were obtained by opening the abdomen and removing approximately $1 \mathrm{~cm}$ of the end section of the cecum, which was stored in a Corning tube. The anatomical and sampling procedures were performed in a biosafety cabinet. All samples were stored at $-80{ }^{\circ} \mathrm{C}$ and prepared for DNA extraction. Detailed information regarding the animals is provided in Table 5 . In this study, NMRs between 10 and 20 weeks and between 20 and 35 weeks were defined as young and adult NMRs, respectively, for comparison of their microbiota.

\section{DNA extraction and PCR amplification}

Bacterial genomic DNA was extracted using E.Z.N.A. Soil DNA Kit (Omega Bio-tek, Inc., Norcross, GA, USA) according to the manufacturer's instructions. The DNA concentration and purity were determined using a Qubit

\begin{tabular}{|c|c|c|c|c|c|}
\hline \multirow{2}{*}{$\begin{array}{l}\text { Age } \\
\text { (week) }\end{array}$} & \multicolumn{2}{|c|}{ 1st collection } & \multicolumn{2}{|c|}{ 2nd collection } & \multirow[t]{2}{*}{ tota } \\
\hline & 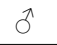 & q & $\hat{0}$ & q & \\
\hline $10-15$ & 9 & 7 & 7 & 6 & 29 \\
\hline $16-20$ & 5 & 6 & 2 & 3 & 16 \\
\hline $21-25$ & 0 & 2 & 0 & 0 & 2 \\
\hline $26-30$ & 2 & 2 & 8 & 2 & 14 \\
\hline $31-35$ & 0 & 0 & 1 & 1 & 2 \\
\hline Total & 16 & 17 & 18 & 12 & 63 \\
\hline
\end{tabular}


2.0 Fluorometer (ThermoFisher Scientific, Waltham, MA, USA). The V3 and V4 hypervariable regions of the bacterial 16S rRNA were amplified using KAPA HiFi Hot Start Ready Mix (2×) (TaKaRa Bio Inc., Kusatsu, Shiga, Japan) and the following pair of $16 \mathrm{~S}$ universal primers: 341F (5'-CCTACGGGNGGCWGCAG-3') and 805R (5'-GACTACHVGGGTATCTAATCC-3') [31]. The reaction was carried out as follows in a final volume of $30 \mu \mathrm{L}: 2 \mu \mathrm{L}$ of microbial DNA $(10 \mathrm{ng} / \mu \mathrm{L})$ as the template, $1 \mu \mathrm{L}$ of each primer $(10 \mu \mathrm{M})$, and $15 \mu \mathrm{L}$ of $2 \times$ KAPA HiFi Hot Start Ready Mix. The PCR was performed using an Applied Biosystems 9700 (ABI, Foster City, CA, USA) with the following program: $95{ }^{\circ} \mathrm{C}$ for 3 min; 5 cycles at $95{ }^{\circ} \mathrm{C}$ for $30 \mathrm{~s}, 45^{\circ} \mathrm{C}$ for $30 \mathrm{~s}$, and $72^{\circ}$ $\mathrm{C}$ for $30 \mathrm{~s} ; 20$ cycles at $95{ }^{\circ} \mathrm{C}$ for $30 \mathrm{~s}, 55{ }^{\circ} \mathrm{C}$ for $30 \mathrm{~s}$, and $72{ }^{\circ} \mathrm{C}$ for $30 \mathrm{~s}$; and a final extension step at $72{ }^{\circ} \mathrm{C}$ for $5 \mathrm{~min}$. The PCR products were evaluated by $1 \%$ $(w / v)$ agarose gel electrophoresis using TBE buffer, staining with ethidium bromide and visualization under ultraviolet light.

\section{S gene library construction, quantification, and sequencing}

After assessment, the PCR products were purified using Agencourt AMPure XP magnetic beads (Beckman Coulter, USA) to remove free primers and primer dimer species from the product. Illumina libraries were constructed using the universal Illumina adaptor and index. Prior to sequencing, the DNA concentration of each amplicon was quantified using a Qubit $^{\circ} 2.0$ BR dsDNA assay kit (ThermoFisher Scientific, Waltham, MA, USA), and quality control was performed using an Agilent 2100 Bioanalyzer (Agilent, Santa Clara, CA, USA). Depending on the coverage needs, all libraries may be pooled for a single run. Each mixture of amplicons was pooled in equimolar ratios based on their concentration. Sequencing was performed using the Illumina MiSeq platform (Illumina MiSeq, San Diego, CA, USA) with the paired-end 300 bp at Sangon BioTech, Co., Ltd., Shanghai, China. Raw reads were deposited in the NCBI Sequence Read Archive (SRA) database under the following accession numbers: SRS2177386 and SRS2177389.

\section{Sequence processing and bioinformatic analysis}

After sequencing, raw reads were assembled using FLASH software (v0.9.6) [32] based on overlap, and fastq files were processed to generate individual fasta and qual files. The paired-end reads in each sample were divided according to unique barcodes. Processing of the assembled sequence reads was performed using the software package MOTHUR (Version 1.30.1) [33] with the following criteria: (1) sequence lengths $<200 \mathrm{bp}$ and $>480$ bp were dislodged; (2) sequences containing ambiguous bases or a homopolymer greater than $6 \mathrm{bp}$ in length were removed [34]; (3) bases with trailing quality scores $<20$ were removed, reads with a 10-bp sliding window were scanned, and truncated reads shorter than $50 \mathrm{bp}$ were discarded; (4) merged reads with a mismatch ratio in overlapping regions >0.1 were removed; and (5) noise was removed using the Pre-cluster tool. High-quality bacterial and archaeal sequences were clustered into OTUs using Usearch (version 5.2.236) with a similarity threshold of 97\% [35]. Chimeric sequences and singleton OTUs were also identified and removed using UCHIME (version 4.2.40) [36]. Taxonomy based on the 16S rRNA gene sequence was assessed using the Ribosomal Database Project (RDP) classifier [37] against the Silva database [38] at a confidence level of 0.8 . Microbial diversity in the individual cecum samples was estimated using rarefaction analysis. Alpha diversity and beta diversity indexes were calculated using MOTHUR [33]. A Bray-Curtis tree of hierarchical clustering was constructed using the unweighted pair group method with arithmetic mean (UPGMA). NMDSs were all conducted using $\mathrm{R}$ software (version 3.2) [39]. Statistical comparisons of the microbiota composition between the first and second sequencing runs were analyzed by an independent-samples t-test using SPSS 19.0. Alpha diversity comparisons of male and female NMRs and young and adult NMRs were calculated via Kruskal-Wallis test analysis using the software LEfSe [40]. If associated sub-groups were observed, the differences between the groups and sub-groups were analyzed by a Wilcoxon rank-sum test. Finally, LDA was used to analyze the effect of these differences between the groups. The level of statistical significance was accepted as $P<0.05$.

\section{Abbreviations \\ COPD: Chronic obstructive pulmonary disease; CRC: Colorectal cancer; FLASH: Fast length adjustment of short reads; LDA: Linear discriminant analysis; LEfSe: Linear discriminant analysis effect size; NMDS: Nonmetric multidimensional scaling; NMR: Naked mole-rat; OUTs: Operational taxonomic units; RDP: Ribosomal Database Project; SRA: Sequence read archive; UPGMA: Unweighted pair group method with arithmetic mean}

\section{Acknowledgments}

We thank Pan Liu and Qiaoyun Xiao for their assistance in maintaining the NMR research facility (Second Military Medical University).

\section{Funding}

The design of the study, sample collection, sequencing, data interpretation and the writing of the manuscript were funded by the Key Projects in the National Science \& Technology Pillar Program (No.2015BAI09B02), data analysis was funded by the National Nature Science Foundation of China (No.31700923), animal breeding for laboratory animalization was funded by the Shanghai Committee of Science and Technology (No.16140900100 and No.17140900200).

\section{Availability of data and materials}

The datasets used and analyzed in the current study are available from the corresponding author upon reasonable request.

Authors' contributions

SC conceived the study. SC and WC designed the study. WS, WY, LL and JC performed breeding for laboratory animalization. JX, JW, YF, RF performed 
sample collection. WY, LL, JC, JW, YF, WS and RF performed sequencing and statistical analysis. BY and ZH supervised the study and interpreted data. JX and WC drafted the manuscript. All authors read and approved the final version of the manuscript.

\section{Ethics approval and consent to participate}

The animal experiments were reviewed and approved by the Institutional Animal Care and Use Committee of the Second Military Medical University.

\section{Consent for publication}

Not applicable.

\section{Competing interests}

The authors declare that they have no competing interests.

\section{Publisher's Note}

Springer Nature remains neutral with regard to jurisdictional claims in published maps and institutional affiliations.

\section{Author details}

${ }^{1}$ Laboratory Animal Centre, Second Military Medical University, No.8 Rd. Panshan, Yangpu District, Shanghai, China. ${ }^{2}$ National Institutes for Food and Drug Control, Institute for Laboratory Animal Resources, No.31 Rd. Huatuo, Daxing District, Beijing, China.

\section{Received: 17 July 2017 Accepted: 6 August 2018}

\section{Published online: 22 August 2018}

\section{References}

1. Buffenstein R. The naked mole-rat: a new long-living model for human aging research. J Gerontol A Biol Sci Med Sci. 2005;60(11):1369-77.

2. Larson J, Park TJ. Extreme hypoxia tolerance of naked mole-rat brain. Neuroreport. 2009;20(18):1634-7.

3. Liang S, Mele J, Wu Y, Buffenstein R, Hornsby PJ. Resistance to experimental tumorigenesis in cells of a long-lived mammal, the naked mole-rat (Heterocephalus glaber). Aging Cell. 2010;9(4):626-35.

4. Park TJ, Lu Y, Juttner R, Smith ES, Hu J, Brand A, Wetzel C, Milenkovic N, Erdmann B, Heppenstall PA, et al. Selective inflammatory pain insensitivity in the African naked mole-rat (Heterocephalus glaber). PLoS Biol. 2008;6(1):e13.

5. Roggenbuck M, Sauer C, Poulsen M, Bertelsen MF, Sorensen SJ. The giraffe (Giraffa camelopardalis) rumen microbiome. FEMS Microbiol Ecol. 2014;90(1):237-46.

6. Wilkinson N, Hughes RJ, Aspden WJ, Chapman J, Moore RJ, Stanley D. The gastrointestinal tract microbiota of the Japanese quail, Coturnix japonica. Appl Microbiol Biotechnol. 2016;100(9):4201-9.

7. Guan Y, Zhang H, Gao X, Shang S, Wu X, Chen J, Zhang W, Jiang M, Zhang $B$, Chen $P$. Comparison of the bacterial communities in feces from wild versus housed sables (Martes zibellina) by high-throughput sequence analysis of the bacterial 16S rRNA gene. AMB Express. 2016;6(1):98.

8. Backhed F, Ley RE, Sonnenburg JL, Peterson DA, Gordon Jl. Host-bacterial mutualism in the human intestine. Science. 2005;307(5717):1915-20.

9. Flint HJ, Scott KP, Louis P, Duncan SH. The role of the gut microbiota in nutrition and health. Nat Rev Gastroenterol Hepatol. 2012;9(10):577-89.

10. Round JL, Lee SM, Li J, Tran G, Jabri B, Chatila TA, Mazmanian SK. The tolllike receptor 2 pathway establishes colonization by a commensal of the human microbiota. Science. 2011;332(6032):974-7.

11. Xu X, Zhang X. Effects of cyclophosphamide on immune system and gut microbiota in mice. Microbiol Res. 2015;171:97-106.

12. Zeng MY, Cisalpino D, Varadarajan S, Hellman J, Warren HS, Cascalho M, Inohara N, Nunez G. Gut microbiota-induced immunoglobulin G controls systemic infection by symbiotic bacteria and pathogens. Immunity. 2016; 44(3):647-58.

13. Zitvogel L, Galluzzi L, Viaud S, Vetizou M, Daillere R, Merad M, Kroemer G. Cancer and the gut microbiota: an unexpected link. Sci Transl Med. 2015; 7(271):271ps271

14. Gao R, Gao Z, Huang L, Qin H. Gut microbiota and colorectal cancer. Eur J Clin Microbiol Infect Dis. 2017;36(5):757-69.

15. Vetizou M, Pitt JM, Daillere R, Lepage P, Waldschmitt N, Flament C, Rusakiewicz S, Routy B, Roberti MP, Duong CP, et al. Anticancer immunotherapy by CTLA-4 blockade relies on the gut microbiota. Science. 2015;350(6264):1079-84.
16. Debebe T, Holtze S, Morhart M, Hildebrandt TB, Rodewald S, Huse K, Platzer M, Wyohannes D, Yirga S, Lemma A, et al. Analysis of cultivable microbiota and diet intake pattern of the long-lived naked mole-rat. Gut Pathog. 2016;8:25.

17. Debebe T, Biagi E, Soverini M, Holtze S, Hildebrandt TB, Birkemeyer C, Wyohannis D, Lemma A, Brigidi P, Savkovic V, et al. Unraveling the gut microbiome of the long-lived naked mole-rat. Sci Rep. 2017;7(1):9590.

18. Yu C, Wang S, Yang G, Zhao S, Lin L, Yang W, Tang Q, Sun W, Cui S. Breeding and rearing naked mole-rats (Heterocephalus glaber) under laboratory conditions. J Am Assoc Lab Anim Sci. 2017;56(1):98-101.

19. Whalen JG, Mully TW, English JC 3rd. Spontaneous Citrobacter freundii infection in an immunocompetent patient. Arch Dermatol. 2007:143(1):124-5

20. Dalben M, Varkulja G, Basso M, Krebs VL, Gibelli MA, van der Heijden I, Rossi F, Duboc G, Levin AS, Costa SF. Investigation of an outbreak of Enterobacter cloacae in a neonatal unit and review of the literature. J Hosp Infect. 2008; 70(1):7-14.

21. Marsland BJ, Yadava K, Nicod LP. The airway microbiome and disease. Chest 2013:144(2):632-7.

22. Gollwitzer ES, Saglani S, Trompette A, Yadava K, Sherburn R, McCoy KD, Nicod LP, Lloyd CM, Marsland BJ. Lung microbiota promotes tolerance to allergens in neonates via PD-L1. Nat Med. 2014;20(6):642-7.

23. Igartua C, Davenport ER, Gilad Y, Nicolae DL, Pinto J, Ober C. Host genetic variation in mucosal immunity pathways influences the upper airway microbiome. Microbiome. 2017:5(1):16.

24. Chen T, Shi Y, Wang X, Meng F, Yang S, Yang J, Xin H. Highthroughput sequencing analyses of oral microbial diversity in healthy people and patients with dental caries and periodontal disease. Mol Med Rep. 2017; 16(1):127-32.

25. Xiao X, Li Y, Zhang G, Gao Y, Kong Y, Liu M, Tan Y. Detection of bacterial diversity in rat's periodontitis model under imitational altitude hypoxia environment. Arch Oral Biol. 2012;57(1):23-9.

26. Man WH, de Steenhuijsen Piters WA, Bogaert D. The microbiota of the respiratory tract: gatekeeper to respiratory health. Nat Rev Microbiol. 2017; 15(5):259-70.

27. Bootz F, Kirschnek S, Nicklas W, Wyss SK, Homberger FR. Detection of Pasteurellaceae in rodents by polymerase chain reaction analysis. Lab Anim Sci. 1998:48(5):542-6.

28. Tap J, Mondot S, Levenez F, Pelletier E, Caron C, Furet JP, Ugarte E, MunozTamayo R, Paslier DL, Nalin R, et al. Towards the human intestinal microbiota phylogenetic core. Environ Microbiol. 2009;11(10):2574-84.

29. Baxter NT, Zackular JP, Chen GY, Schloss PD. Structure of the gut microbiome following colonization with human feces determines colonic tumor burden. Microbiome. 2014:2:20.

30. Chen ZY, Hsieh YM, Huang CC, Tsai CC. Inhibitory Effects of Probiotic Lactobacillus on the Growth of Human Colonic Carcinoma Cell Line HT-29. Molecules. 2017;22(1):1-12.

31. Herlemann DP, Labrenz M, Jurgens K, Bertilsson S, Waniek JJ, Andersson AF. Transitions in bacterial communities along the $2000 \mathrm{~km}$ salinity gradient of the Baltic Sea. ISME J. 2011;5(10):1571-9.

32. Magoc T, Salzberg SL. FLASH: fast length adjustment of short reads to improve genome assemblies. Bioinformatics. 2011;27(21):2957-63.

33. Schloss PD, Westcott SL, Ryabin T, Hall JR, Hartmann M, Hollister EB, Lesniewski RA, Oakley BB, Parks DH, Robinson CJ, et al. Introducing mothur: open-source, platform-independent, community-supported software for describing and comparing microbial communities. Appl Environ Microbiol. 2009;75(23):7537-41.

34. Kochling T, Sanz JL, Gavazza S, Florencio L. Analysis of microbial community structure and composition in leachates from a young landfill by 454 pyrosequencing. Appl Microbiol Biotechnol. 2015;99(13):5657-68.

35. Edgar RC. Search and clustering orders of magnitude faster than BLAST. Bioinformatics. 2010;26(19):2460-1.

36. Edgar RC, Haas BJ, Clemente JC, Quince C, Knight R. UCHIME improves sensitivity and speed of chimera detection. Bioinformatics. 2011;27(16):2194-200.

37. Cole JR, Wang Q, Cardenas E, Fish J, Chai B, Farris RJ, Kulam-Syed-Mohideen AS, McGarrell DM, Marsh T, Garrity GM, et al. The ribosomal database project: improved alignments and new tools for rRNA analysis. Nucleic Acids Res. 2009;37(Database issue):D141-5.

38. Quast C, Pruesse E, Yilmaz P, Gerken J, Schweer T, Yarza P, Peplies J Glockner FO. The SILVA ribosomal RNA gene database project: improved 
data processing and web-based tools. Nucleic Acids Res. 2013;41(Database issue):D590-6.

39. Development Core Team R. R: a Language and Environment for Statistical Computing. Computing. 2011;14:12-21.

40. Segata N, Izard J, Waldron L, Gevers D, Miropolsky L, Garrett WS, Huttenhower C. Metagenomic biomarker discovery and explanation. Genome Biol. 2011;12(6):R60.

Ready to submit your research? Choose BMC and benefit from:

- fast, convenient online submission

- thorough peer review by experienced researchers in your field

- rapid publication on acceptance

- support for research data, including large and complex data types

- gold Open Access which fosters wider collaboration and increased citations

- maximum visibility for your research: over $100 \mathrm{M}$ website views per year

At $\mathrm{BMC}$, research is always in progress.

Learn more biomedcentral.com/submissions 